\title{
3,4-Di-O-Caffeoylquinic Acid Inhibits Angiotensin-II-Induced Vascular Smooth Muscle Cell Proliferation and Migration by Downregulating the JNK and PI3K/Akt Signaling Pathways
}

\author{
Wen-Fei Chiou, ${ }^{1,2,3}$ Chien-Chih Chen, ${ }^{1}$ and Bai-Luh Wei ${ }^{2}$ \\ ${ }^{1}$ National Research Institute of Chinese Medicine, Taipei 112, Taiwan \\ ${ }^{2}$ Institute of Life Science, Collage of Science and Engineering, National Taitung University, Taitung, Taiwan \\ ${ }^{3}$ Institute of Traditional Medicine, School of Medicine, National Yang-Ming University, Taipei, Taiwan
}

Correspondence should be addressed to Wen-Fei Chiou,wfchiou@cma23.nricm.edu.tw

Received 14 May 2009; Accepted 17 August 2009

Copyright (c) 2011 Wen-Fei Chiou et al. This is an open access article distributed under the Creative Commons Attribution License, which permits unrestricted use, distribution, and reproduction in any medium, provided the original work is properly cited.

\begin{abstract}
We previously reported 3,4-di-O-caffeoylquinic acid (CQC) protected vascular endothelial cells against oxidative stress and restored impaired endothelium-dependent vasodilatation. Here, we further investigated its anti-atherosclerotic effect against angiotensin II (Ang II) evoked proliferation and migration of cultured rat vascular smooth muscle cells (rVSMC). The results showed CQC $(1-20 \mu \mathrm{M})$ clearly inhibited Ang-II-stimulated BrdU incorporation and cell migration of rVSMC in a concentrationdependent manner but without significant cytotoxicity. Western blot analysis revealed Ang II increased the phosphorylation levels of Akt and mitogen-activated protein kinases (MAPKs;p38, ERK1/2 and JNK) in rVSMC. In the presence of phosphatidylinositol 3-kinase (PI3K) inhibitor wortmannin and three individual MAPK inhibitors SB203580, PD98059 and SP600125, both Ang-IIinduced cell proliferation and migration were significantly attenuated, although to differing extents, suggesting the PI3K and MAPK signal pathways all participated in regulating rVSMC proliferation and migration. Also, the CQC pretreatment markedly suppressed Ang-II-induced phosphorylation of Akt and JNK rather than ERK1/2, although it failed to affect p38 phosphorylation. In conclusion, our data demonstrate CQC may act by down-regulating Akt, JNK and part of the ERK1/2 pathways to inhibit Ang-II-induced rVSMC proliferation and migration. The anti-atherosclerotic effect of CQC is achieved either by endothelial cells protection or by VSMC proliferation/migration inhibition, suggesting this compound may be useful in preventing vascular diseases.
\end{abstract}

\section{Introduction}

Migration and proliferation of vascular smooth muscle cells (VSMCs) are critical events in the development of restenosis and in the progression of atherosclerosis [1]. A growing body of evidence supports the finding that angiotensin II (Ang II) plays an important role in the pathogenesis of several cardiovascular diseases associated with VSMC proliferation and migration. Ang II also promotes the generation of oxidative stress in the vasculature, appearing to be a key mediator of Ang-II-induced VSMC proliferation and migration, endothelial cell apoptosis and endothelial dysfunction, lipoprotein peroxidation and adhesion molecules expression, all of which participate in the induction of atherosclerosis.

Different signal transduction cascades have been implicated in Ang-II-mediated cell growth and migration, such as the mitogen-activated protein kinase (MAPK) pathways
[2]. Furthermore, reactive oxygen species (ROS) generation in response to Ang II stimuli has been shown to relate to the activation of MAPKs and induces proliferation of VSMCs [3]. On the other hand, Ang II has been shown to activate phosphatidylinositol 3-kinases (PI3Ks) in the cultured porcine carotid artery VSMC [4]. PI3K play a major role in a wide range of cellular processes, including motility and cell cycle progression [5]. Recent evidence suggests the PI3K pathway is implicated in VSMC mitogenesis. Shigematsu et al. [6] showed activating PI3K is essential for initiating medial VSMC replication after balloon injury in rats. In addition, the direct role of PI3K in Ang-II-induced DNA synthesis and proliferation has been described in cultured porcine carotid artery VSMC [4]. One of the major downstream targets of PI3K is Akt. The results obtained from Dugourd et al. [7] provide the first evidence of a specific and necessary role of Akt in Ang-II-induced proliferation. 


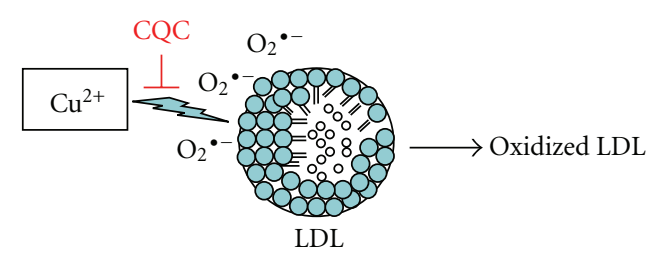

(a)

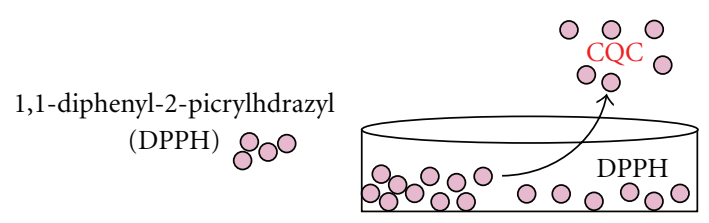

(b)

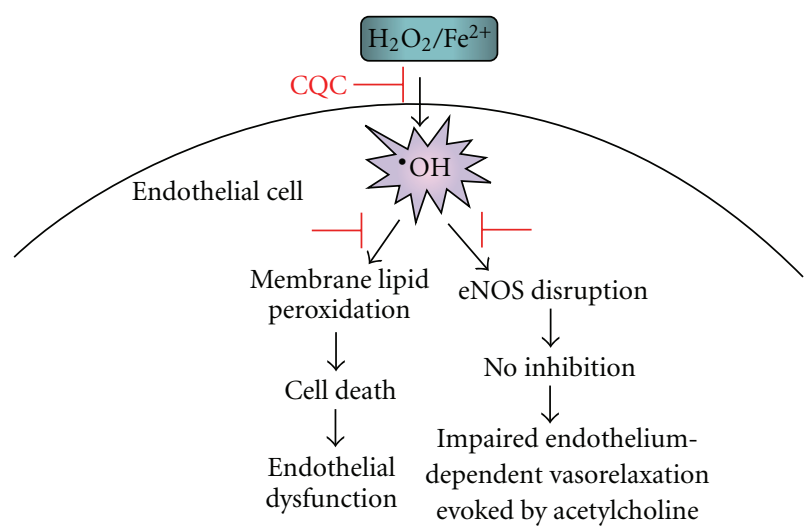

(c)

FIgURE 1: Various anti-oxidative properties by CQC. In cell free system, CQC displayed potent antioxidant activity to inhibit $\mathrm{Cu}^{2+}$ induced oxidation of human LDL (a) and scavenge DPPH free radical (b). In cultured HUVECs, CQC minimized not only the loss of cell viability induced by oxidative stress but also the ROS-induced impairment of endothelium-dependent relaxation to acetylcholine in rat aorta (c).

Antioxidants such as resveratrol possess many biologic activities including protection from or reduction of the incidence of coronary heart disease [8] and a direct relaxant effect on the vascular smooth muscle [9]. Chao et al. also reported resveratrol can inhibit Ang-II-induced proliferation in rat aortic smooth muscle cells [10]. We previously demonstrated 3,4-di-O-caffeoylquinic acid (CQC), a phenolic constituent isolated from a Taiwanese folk medicine Elephantopus mollis, minimized not only the loss of cell viability induced by oxidative stress in cultured human umbilical vein endothelial cells (HUVECs) but also the ROSinduced impairment of endothelium-dependent relaxation to acetylcholine in rat aorta through an antioxidant property (Figure 1(c)) [11]. However, whether CQC helps prevent VSMCs from atherosclerotic damage remains unclear. In the present study, we investigate the effect of CQC on Ang-IIinduced migration and proliferation of rat cultured VSMC and further delineated the possibly involved action mechanisms.

\section{Methods}

2.1. Materials. 3,4-Di-O-caffeoylquinic acid (CQC) was isolated by our own laboratory [11]. Its purity determined by high-performance liquid chromatography with an ultraviolet detector was between 99.1 and $99.8 \%$. This drug was first dissolved in dimethylsulphoxide at $100 \mathrm{mM}$ and then serially diluted in PBS immediately prior to experiments. Stock solution of CQC was used within 1 week after preparation. For examination of the effect of CQC, $10 \mu \mathrm{l}$ of drug solution was added to $1 \mathrm{ml}$ culture medium. Ang II, SB203580, PD 98059 and SP600125 were obtained from Sigma-Aldrich (St. Louis, MO, USA). Wortmannin was procured from Calbiochem (Merck Ltd, Taiwan). Alamar Blue Assay kit and BrdU cell proliferation assay kit was purchased from Serotec (Oxford, UK) and Chemicon (Millipore, MA, USA), respectively. Dulbecco's modified Eagle's medium (DMEM) was purchased from GIBCO/BRL (Grand Island, NY 14072, USA). Fetal bovine serum (FBS), penicillin, streptomycin and trypsin/EDTA were obtained from Biological Industries (Kibbutz Beit Haemek 25115, Israel). VSMC-specific goat anti-actin and HRP anti-rabbit antibody were purchased from Santa Cruz (Santa Cruz, CA, USA). Antibodies against total (i.e., inactivated) and the phosphorylated (i.e., activated) forms of p38, ERK1/2, JNK and Akt were purchased from Cell Signaling Technology (Beverly, MA, USA). ECL reagent was obtained from Amersham Pharmacia (Buckinghamshire, UK).

2.2. Culture of Rat Vascular Smooth Muscle Cells (rVSMC). The investigation was approved by the IACUC of National Research Institute of Chinese Medicine. VSMC were isolated from the thoracic aorta of 6- to 8-week-old male SpragueDawley rats by explant culture. Subcultures were prepared by dissociation of cells with trypsin/EDTA (Sigma-Aldrich) and seeding into appropriate culture dishes and grown until approximately $80 \%$ confluent in DMEM containing $10 \%$ FBS, $100 \mu \mathrm{g} / \mathrm{ml}$ penicillin and $100 \mathrm{U} / \mathrm{ml}$ streptomycin (complete medium) under standardized culture condition. Cells were identified as VSMC by their characteristic morphology as well as with antibodies to VSMC-specific goat anti-actin and were used from passage 3 to 10 . VSMCs were grown in complete medium until $12 \mathrm{~h}$ prior to experimentation, at which time cells were incubated in low serum medium $(0.3 \%$ FCS $)$ for all experiments. Cells were then preincubated with different concentrations of CQC for $30 \mathrm{~min}$ and then stimulated with or without Ang II for different incubation times as indicated, followed by proliferation assay or harvesting for western blot analysis. Cellular viability was determined by Alamar Blue assay.

2.3. Cell Viability Measurement. Cell viability was monitored by Alamar Blue Assay kit as described previously [12]. After incubation with or without CQC, Alamar Blue growth indicator dye $(10 \%, \mathrm{v} / \mathrm{v})$ was added to cell culture for another $4 \mathrm{~h}$ incubation at $37^{\circ} \mathrm{C}$. The change in colour could be monitored with an ELISA reader at $620 \mathrm{~nm}$. Wells containing medium and Alamar Blue dye without cells were 
used as blanks. All experiments were performed in duplicate and repeated at least twice with similar results. The mean absorbance for the duplicate cultures of each drug was calculated and the mean blank value was subtracted from these. Cell viability in control medium without any treatment was represented as $100 \%$.

2.4. Cell Proliferation Analysis. rVSMCs proliferation was measured by using the BrdU cell proliferation assay kit according to the manufacturer's protocol. In brief, rVSMCs were grown in low serum medium $(0.3 \%$ FCS $)$ for $12 \mathrm{~h}$ to induce cell growth arrest. After the quiescent cells were pretreated with or without different concentrations of tested drugs (CQC or protein kinase inhibitors) for $30 \mathrm{~min}$, Ang II were added to the medium and incubated for further $48 \mathrm{~h}$. After that, BrdU was added and the cells were reincubated for $4 \mathrm{~h}$. After removing the culture medium, rVSMCs were fixed and the DNA denatured. Then, peroxidase-labelled anti-BrdU was added to bind to the BrdU. The immune complexes were detected by the 3,3',5,5'-tetramethyl-benzidine substrate reaction, and the resultant color was read at $450 \mathrm{~nm}$ in a microplate spectrophotometer. The absorbance values correlated directly to the amount of DNA synthesis and thereby to the number of proliferating cells in culture.

2.5. Migration Assay. Cell migration was assessed using a 24well Transwell with a membrane pore size of $5 \mu \mathrm{m}$ (Corning Costar, NY, USA) as previously described [12]. Briefly, $90 \mu \mathrm{l}$ of $\operatorname{rVSMC}\left(1.0 \times 10^{6} \mathrm{ml}^{-1}\right)$ were added to each of the upper wells in the presence of $10 \mu \mathrm{l}$ PBS or drugs (CQC or protein kinase inhibitors) for $30 \mathrm{~min}$, respectively. The lower well was filled with $600 \mu \mathrm{l}$ of serum-free medium that contained $100 \mathrm{nM}$ Ang II to assess the migration activity. After incubation for $8 \mathrm{~h}$ at $37^{\circ} \mathrm{C}$ in a $5 \% \mathrm{CO}_{2}$ incubator, filters were cut and the cells on the top surface of the membrane were removed with a cotton swab. The cells that had migrated to the lower side of the filter were fixed, stained with Haematoxylin solution and counted under a microscope (magnification, $\times 400$ ) for quantification of rVSMC migration [13]. Migration activity was calculated as the mean number of migrated cells observed in four high-power fields and given as the mean value of four measurements. In the experiment that presence of protein kinase inhibitors, Ang-II- induced migration was designated as $100 \%$ and results were represented as relative migration.

2.6. Cell Extracts Preparation and Western Blot Analysis. After incubation, cell pellets were lysed with ice-cold lysis buffer and centrifuged at $45000 \mathrm{~g}$ for $1 \mathrm{~h}$ at $4^{\circ} \mathrm{C}$ to yield the whole cell extract in the supernatants [12]. Protein concentration was determined using BCA reagents (Pierce, USA) according to the manufacturer's manual. Protein $(40 \mu \mathrm{g})$ was separated using $8 \%$ SDS-polyacrylamide gel and transferred to a nitrocellulose membrane. After blocking, the membrane was incubated (overnight at $4^{\circ} \mathrm{C}$ ) with antibodies that specifically detect the total and the phosphorylated forms of p38, ERK1/2, JNK and Akt, respectively. Then, it was incubated with HRP anti-rabbit antibody and detected by ECL. The results were evaluated by densitometry analysis.

2.7. Statistics. All values in the text and figures represent mean \pm SE. Statistical significance was determined using a two-tailed Student $t$-test. Statistical significance with $P$ values of $<.01$ or $<.001$ were designated with a single “ $*$ " or double “**”, respectively.

\section{Results}

3.1. CQ Inhibited Ang-II-Induced Proliferation of rVSMC. The results shown in Figure 2(a) indicated growth-arrested rVSMCs treated with Ang II (1-1000 nM) significantly increased BrdU incorporation in a concentration-dependent manner. Compared to the control group, $100 \mathrm{nM}$ of Ang II treatment increased cellular proliferation by about $200 \%$. In the presence of CQC $(1,5,10$ and $20 \mu \mathrm{M}), 100 \mathrm{nM}$ AngII-stimulated cell proliferation was significantly inhibited in a concentration-dependent manner (Figure 2(b)). Cell viability assay indicated none of the concentrations used for CQC displayed significant cytotoxicity: cell viability in the presence of $20 \mu \mathrm{M}$ CQC in rVSMCs was greater than $92 \pm$ $5 \%$.

3.2. Ang-II-Induced Migration of rVSMC Was Suppressed by $C Q C$. The stimulatory effect of Ang II on the migration of rVSMC is shown in Figure 3(a). As expected, Ang II $(1-1000 \mathrm{nM})$ induced cell migration in a concentrationdependent manner. The increase in migration activity was maximal at the concentration of $100 \mathrm{nM}$ Ang II. In the presence of CQC, such migration was significantly and dosedependently inhibited (Figure 3(b)).

\begin{abstract}
3.3. Protein Kinases Phosohorylation in Response to Ang II Stimulation. It has previously been reported Ang-II-induced signalling in VSMC involves the activation of multiple protein kinases. Thus, we measured Ang-II-induced kinase phosphorylation in rVSMCs. When cells were stimulated with Ang II, the phosphorylation of the three MAPKs were all significantly induced as early as $10 \mathrm{~min}$ after the stimulation and lasted up to $60 \mathrm{~min}$, and diminishing after that (data not shown). Thus, the $10 \mathrm{~min}$ incubation period was chosen to assess the concentration-dependent effect of Ang II. Figure 4(a) shows the phosphorylation of p38, ERK1/2 and JNK in rVSMCs was increased after being stimulated with Ang II (1-100 nM) for $10 \mathrm{~min}$, reaching a maximal response at $100 \mathrm{nM}$ Ang II $(n=4)$. Western blots probed for Akt (a downstream target for PI3K) phosphorylation at the Serine 473 site were analyzed as a measure of Akt activation. The phosphorylation of Akt also showed concentrationdependent increases and was consistent with MAPK phosphorylation stimulated by Ang II.
\end{abstract}

3.4. Ang-II-Induced Proliferation and Migration of rVSMCs Were Suppressed by MAPK and PI3K Inhibitors. To clarify whether the Ang-II-induced proliferation or migration 


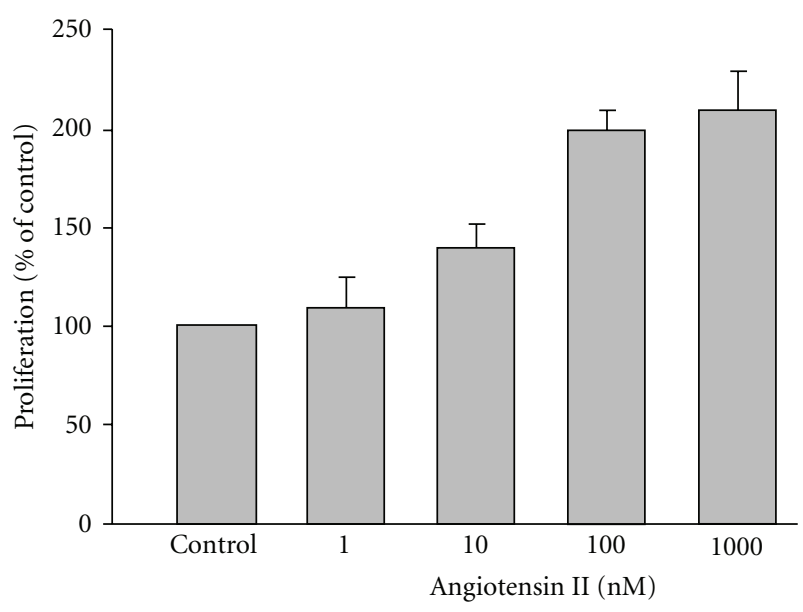

(a)

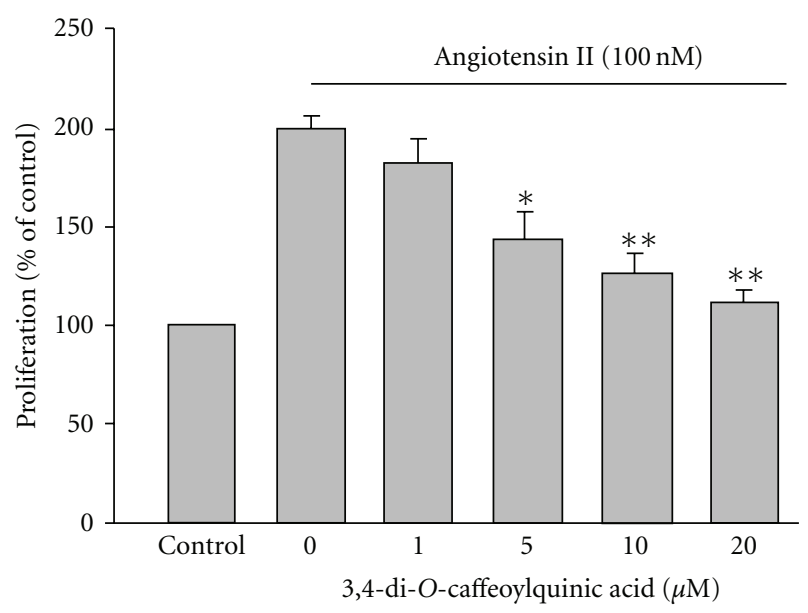

(b)

Figure 2: rVSMC proliferation. (a) Concentration-dependent responsiveness of Ang-II-induced rVSMC proliferation. (b) 3,4-di$O$-caffeoylquinic acid dose-dependently inhibited $100 \mathrm{nM}$ Ang-IIinduced proliferation. ${ }^{*} P<.01$ and ${ }^{* *} P<.001$, significant difference versus samples treated with Ang II alone. Each histogram represents the mean $\pm \mathrm{SE}$ of three separate experiments run in triplicate on four separate cultures.

is really mediated by the MAPK or PI3K signal pathways, rVSMCs were pretreated with p38 MAPK inhibitor SB203580 $(10 \mu \mathrm{M})$, MEK1/2 inhibitor PD98059 $(10 \mu \mathrm{M})$, JNK inhibitor SP600125 $(10 \mu \mathrm{M})$ or PI3K inhibitor wortmannin $(100 \mathrm{nM})$ for 30 min then stimulated with $100 \mathrm{nM}$ Ang II for subsequent migration or proliferation assay, respectively. Figures $4(\mathrm{~b})$ and 4(c) show stimulation with Ang II caused a $217 \pm 12$ and a $327 \pm 19 \%$ significant increase in the proliferation and migration of rVSMC, respectively, compared to the control levels $(=100 \%)$. Ang-II-induced proliferation of rVSMC was significantly suppressed by all protein kinase inhibitors treatment. The results showed SB203580, PD98059 and wortmannin almost eliminated the Ang II effect on proliferation. SP600125 also significantly inhibited such proliferation, although to a lesser extent than inhibition by the others. In the case of migration,

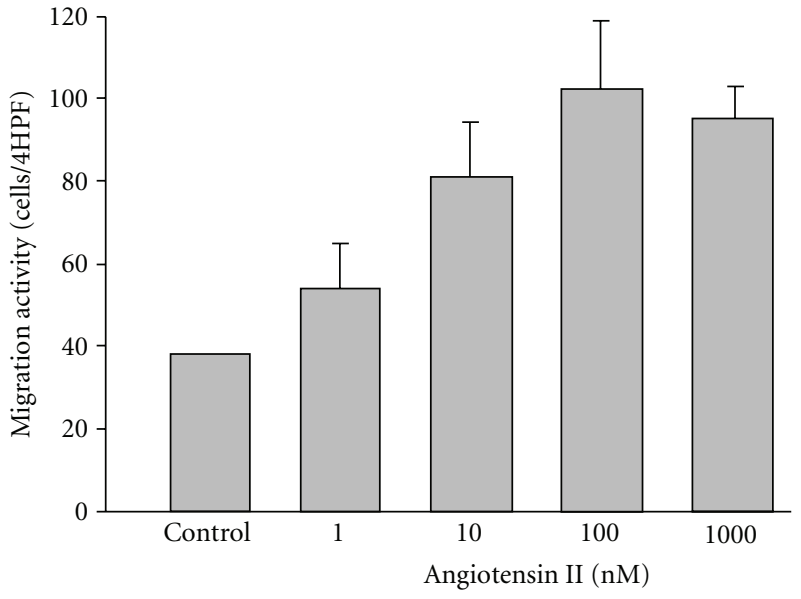

(a)

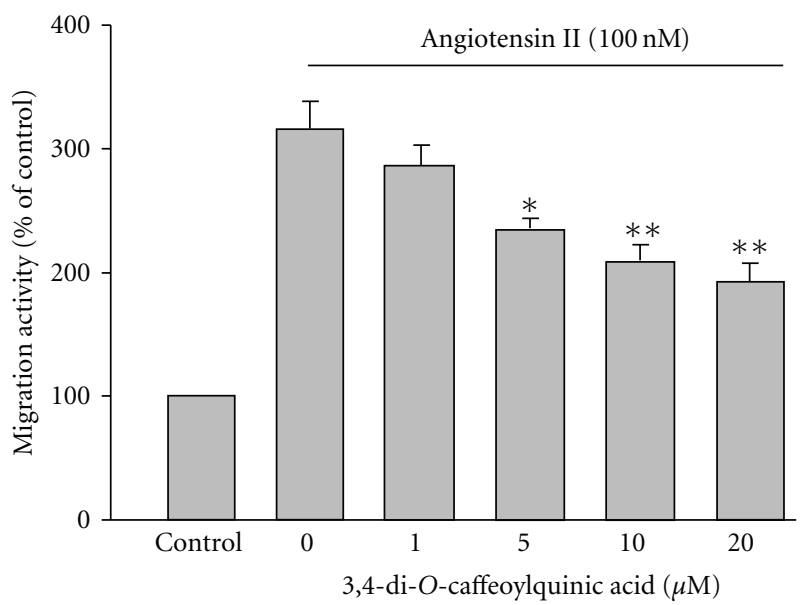

(b)

Figure 3: rVSMC migration. (a) Concentration-dependent response of Ang-II-induced rVSMC migration. (b) 3,4-di-O-caffeoylquinic acid dose-dependently inhibited $100 \mathrm{nM}$ Ang-II-induced migration. ${ }^{*} P<.01$ and ${ }^{* *} P<.001$, significant difference versus samples treated with Ang II alone. Each histogram represents the mean \pm SE of three separate experiments run in triplicate on four separate cultures.

pretreatment with SB203580 and wortmannin abrogated the Ang II effect. SP600125 also modestly reduced Ang-IIinduced migration; however, PD98059 failed to affect such a response (Figure 3(c)). These results indicated p38, ERK1/2, JNK as well as PI3K activation may all be essential for AngII-stimulated proliferation in VSMCs. Nevertheless, p38 and PI3K rather than JNK and ERK1/2 were shown to play more important roles in mediating Ang-II-stimulated migration in rVSMCs.

3.5. CQC Significantly Attenuated ERK1/2, JNK and Akt Phosphorylation Induced by Ang II. To demonstrate whether CQC inhibited Ang-II-induced rVSMC proliferation or migration by affecting the activation of MAPK signals, we analyzed the phosphorylation levels of p38, ERK1/2 and JNK in response 


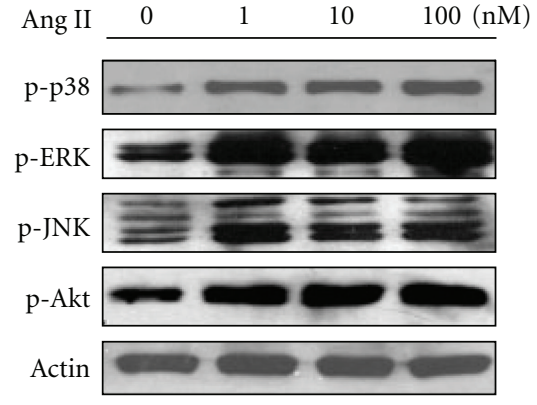

(a)

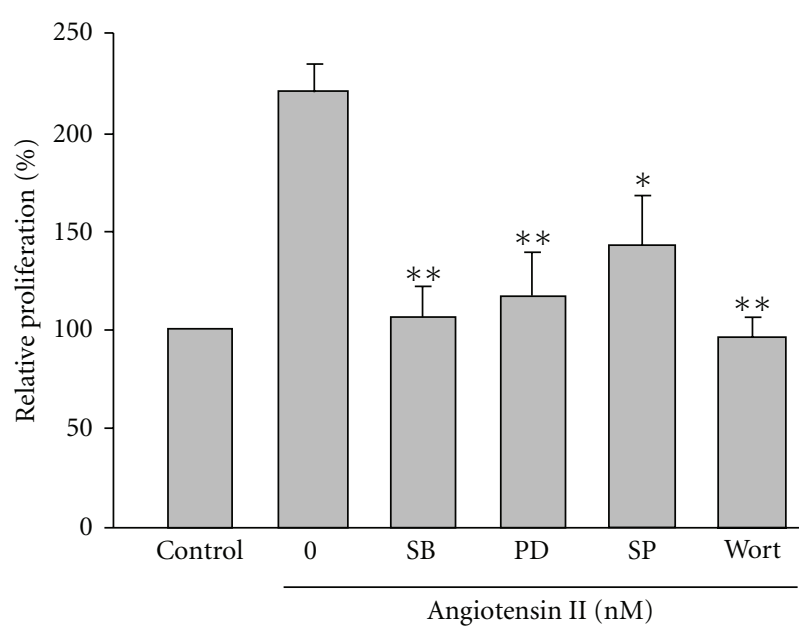

(b)

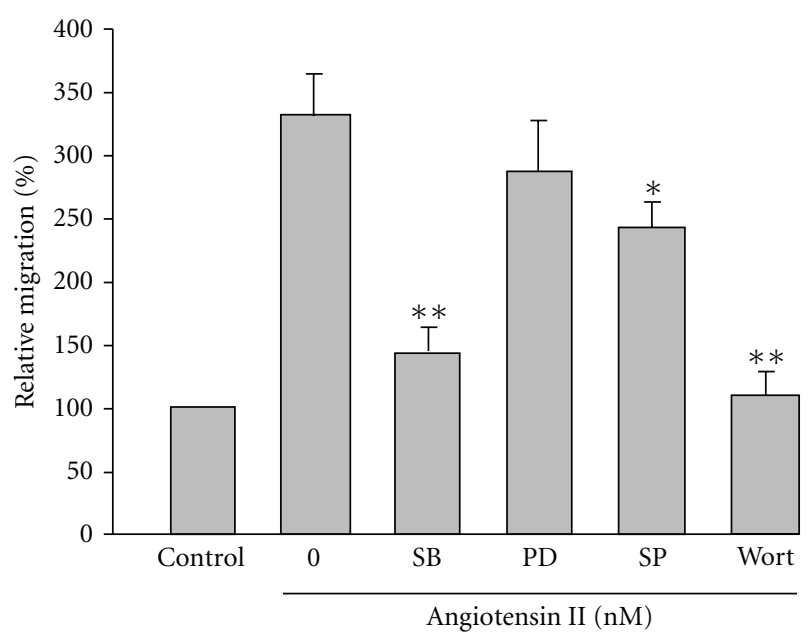

(c)

FIGURE 4: Ang-II induced protein kinses phosphorylation, proliferation and migration. (a) Concentration-dependent responsiveness of Ang-II-induced phosphorylation of p38, ERK1/2, JNK and Akt. (b) Effects of SB203580, PD98059, SP600125 and wortmannin on the Ang-II-induced rVSMC proliferation. (c) Effects of SB203580, PD98059, SP600125 and wortmannin on the Ang-II-induced rVSMC migration. ${ }^{*} P<.01$ and ${ }^{* *} P<.001$, significant difference versus samples treated with Ang II alone. Each histogram represents the mean \pm SE of three separate experiments run in triplicate on three separate cultures. to Ang II after CQC treatment. The results showed Ang-IIstimulated increases of ERK1/2 and JNK phosphorylation were inhibited by this drug in a concentration-dependent manner, respectively (Figure 5(a)). Significant reduction of JNK phosphorylation began at $5 \mu \mathrm{M}$ CQC pretreatment, while ERK1/2 phosphorylation was significantly attenuated in the presence of $10 \mu \mathrm{M}$ CQC. In contrast, Ang-II-evoked phosphorylation of p38 MAPK was not reduced by CQC treatment. On the other hand, CQC at a concentration of $5 \mu \mathrm{M}$ clearly, and at a concentration of $10 \mu \mathrm{M}$ completely, suppressed the effect of Ang II on Akt phosphorylation (Figure 5(b)). These results indicated the effects of CQC on Ang-II-induced proliferation/migration in rVSMCs may be mediated by a coordinated inhibition of the ERK1/2, JNK and Akt signalling pathways.

\section{Discussion}

Ang II acting through the AT1 receptor to mediate VSMC proliferation and migration are important events in the formation of the neointima in pathological states such as atherosclerosis and hypertension [14]. Thus, inhibition of VSMC proliferation or migration represents a potentially important therapeutic strategy for treating such diseases. In this paper, our data demonstrated CQC significantly inhibited not only Ang-II-induced proliferation but also cell migration of rVSMC in a concentration-dependent manner. These results indicated CQC may be a potential pharmaceutical to prevent atherosclerosis.

Many of the signalling events relevant to cell proliferation are mediated through activation of transcription factors by MAPKs, which can be upregulated by Ang II $[2,15]$. Our results support the above idea, since Ang II really upregulated the phosphorylation of p38, ERK1/2 and JNK in rVSMCs (Figure 4(a)) and Ang-II-induced cell proliferation was abrogated in the presence of three individual MAPK inhibitors (Figure 4(b)). We also found CQC significantly inactivated JNK phosphorylation on Ang-II-stimulated rVSMC at a concentration of $5 \mu \mathrm{M}$, but its effect on ERK1/2 phosphorylation was observed at a relatively high concentration of $10 \mu \mathrm{M}$. In contrast, Ang-II-induced phosphorylation of p38 was not nearly as affected by this compound. Therefore, the antiproliferative property of CQC on $\mathrm{rVSMC}$ was associated with the downregulation of JNK rather than the ERK1/2 pathway.

PI3Ks also play a major role in a wide range of cellular processes, including motility and cell cycle progression [5]. Recent evidence suggests the PI3K pathway is implicated in VSMC proliferation. Shigematsu et al. showed activating $\mathrm{PI} 3 \mathrm{~K}$ is essential for initiating medial VSMC replication after balloon injury in rats [6]. In addition, a direct role of PI3K in Ang II-induced DNA synthesis and proliferation has been described in cultured porcine carotid artery VSMC [4]. One of the major downstream targets of PI3K is serine/threonine kinase Akt (also known as protein kinase B). Once phosphorylated, Akt activates various proteins involved in many cellular responses, including cell survival and growth promotion [16]. It has recently been shown 

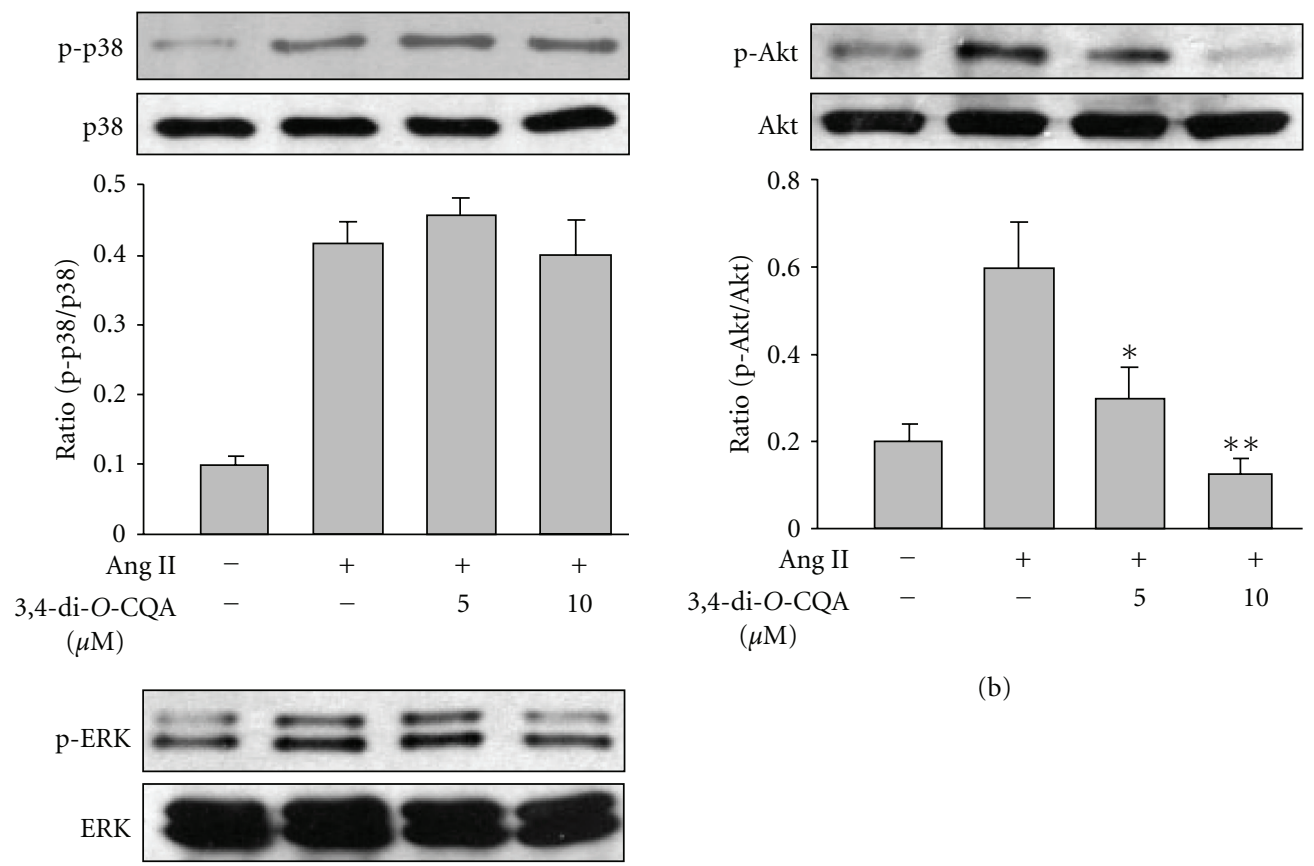

(b)
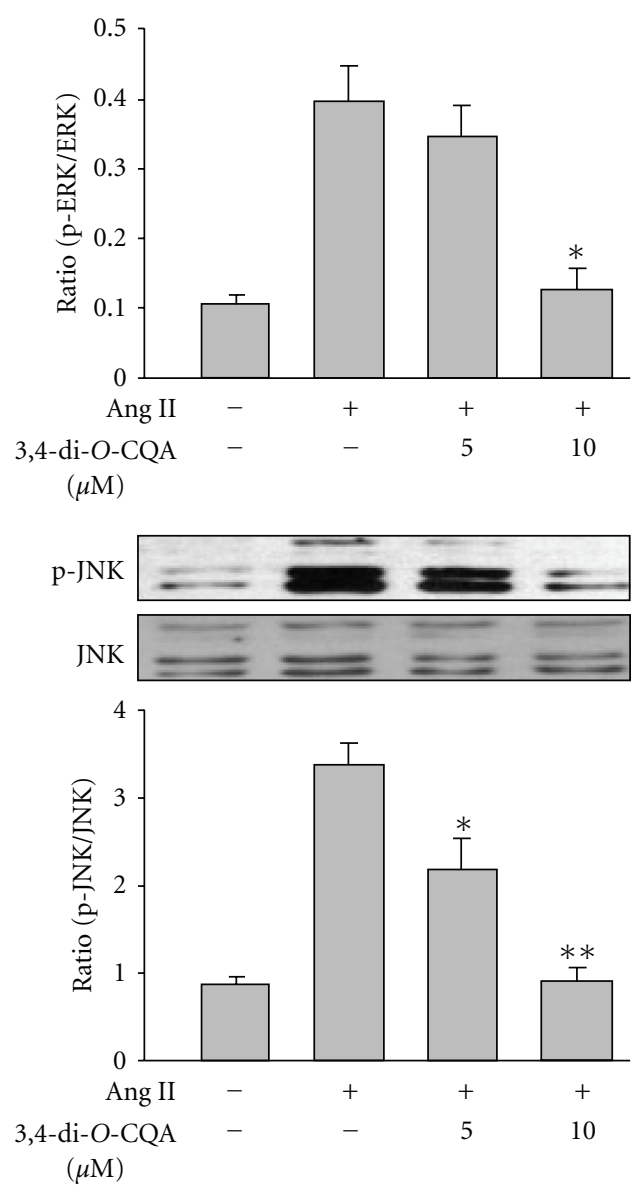

(a)

Figure 5: Effects of 3,4-di-O-caffeoylquinic acid on the Ang-II-induced phosphorylation of p38, ERK1/2, JNK and Akt, respectively. Representative results of the western blots are shown in the top panels, and the data in the bottom panels represent the mean \pm SE of four separate experiments. ${ }^{*} P<.01$ and ${ }^{* *} P<.001$, significant difference versus samples treated with Ang II alone. 


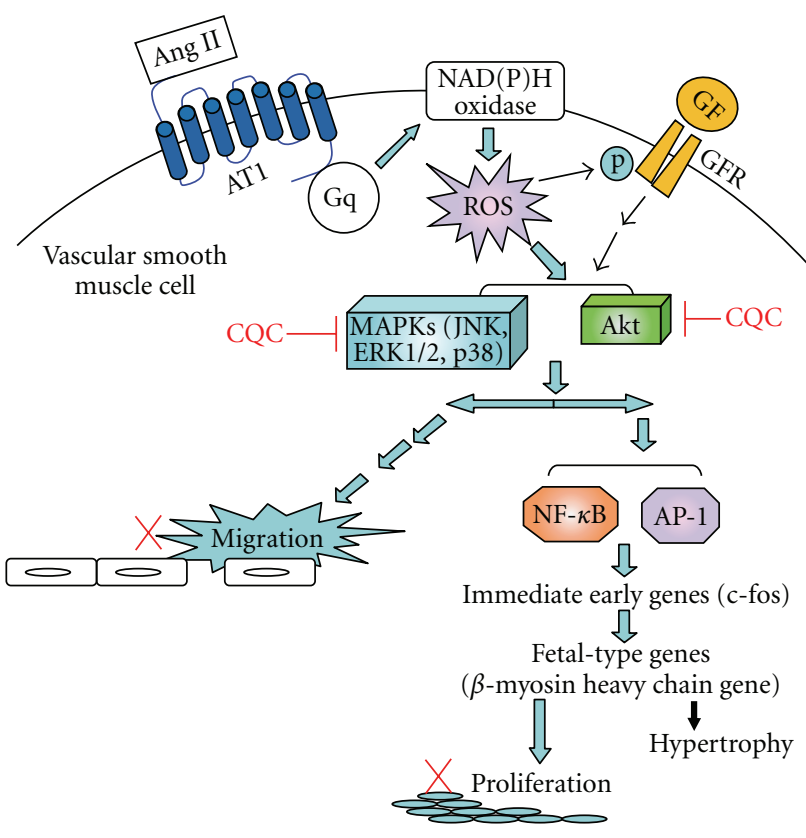

Figure 6: Proposed molecular target of CQC to inhibit Ang-IIinduced proliferation and/or migration of rVSMC. Arrows indicate the main biological end points preceding cell proliferation and migration in response to Ang II. ROS activate hypertrophy and proliferation in VSMC. In response to the growth factors Ang II or PDGF, ROS are endogenously produced by VSMC and stimulate MAPKs and Akt. Our data documented that CQC inhibited Ang-II-induced rVSMC proliferation and migration. The anti-atherosclerotic property of CQC may act by downregulating the Akt, JNK and in part of ERK1/2 pathways by Ang II. AT1: angiotensin type 1 receptor; GFR: growth factor receptor; Gq: Gq protein.

Akt is phosphorylated and activated by the AT1 receptor in a PI3K-dependent manner in various cell types including VSMC $[17,18]$. Dugourd et al. provided a direct role of Akt in Ang II-mediated cell proliferation in rat aortic SMC and suggested Akt stimulation by the AT1 receptor takes place downstream of PI3K activation [7]. We also reconfirmed the important role of Akt in the Ang-II-induced proliferation of rVSMC based on the observations Ang-II-evoked a significant phosphorylation of Akt and Ang-II-induced cell proliferation was wortmannin sensitive. The present results demonstrated CQC clearly blocked Ang-II-activated phosphorylation of Akt suggesting interruption of the PI3K/Akt signal pathway also participated in antiproliferative activity by CQC.

Cell migration is mediated by intracellular kinases including Src, focal adhesion kinase and PI3K, and MAPK is a downstream target of all these kinases [19]. After performing the functional assay using individual kinases inhibitors, our results indicated the $\mathrm{p} 38$ and $\mathrm{PI} 3 \mathrm{~K}$ signal pathways, but not ERK1/2 pathway, were heavily involved in Ang-II-induced migration of rVSMC (Figure 4(c)). Phosphorylation of JNK also played a role in mediating rVSMC migration, however, to a lesser extent. The results obtained here concluded CQC displayed antimigration activity either by inactivating the MAPK signal pathway, particularly JNK, or by downregulation of the PI3K/Akt pathway stimulated by Ang II.

Mounting evidence suggests Ang II activates the NADPH oxidase system and promotes the generation of ROS in vitro, ex vivo and in vivo [20]. Viedt et al. [21] reported the radical species scavenger $\mathrm{N}$-acetyl cysteine or the inhibitor of NADPH oxidase antagonizes the stimulatory effects of Ang II on MAPK activity. These suggested ROS acts as a scaffold molecule linking the signal network between Ang II and MAPKs. In fact, we previously found CQC displayed antioxidant activity more potent than resveratrol in chelating the 1,1-diphenyl-2-picrylhydrazyl (DPPH) free radical and inhibiting $\mathrm{Cu}^{2+}$-induced oxidation of human low-density lipoprotein (LDL; Figures 1(a) and 1(b)) [11]. Furthermore, this compound not only effectively minimized the loss of cell viability induced by Fenton's reagent in cultured HUVEC but also significantly reversed $\mathrm{H}_{2} \mathrm{O}_{2} / \mathrm{FeSO}_{4}$-induced impairment of endothelium-dependent relaxation to acetylcholine in rat aorta (Figure 1(c)). These data suggested CQC prevents cells from undergoing oxidative stress and its scavenging of free radicals could be the key mechanism contributing to the cytoprotective effect of CQC.

Several biological activities, such as the neuroprotective $[22,23]$, analgesic [24] and antioxidant [25, 26] properties have been reported for caffeoylquinic acid or CQC. However, this is the first paper demonstrating CQC can inhibit AngII-induced rVSMC proliferation and migration, probably through downregulating the Akt, JNK and part of the ERK1/2 pathways, in which the anti-oxidant property plays an important role. The proposed molecular targets of CQC to inhibit Ang-II-induced proliferation and/or migration of VSMC are summarized in Figure 6. Hung et al. [27] examined the antioxidant activity of six caffeoylquinic acid derivatives (including CQC) from the roots of Dipsacus asper Wall. They reported these compounds were potent scavengers of DPPH and had the ability to inhibit LDL oxidation as well as the thiobarbituric acid reactive substances formation. Based on these finding, Hung et al. suggested caffeoylquinic acid derivatives may have a role to play in preventing the development and progression of atherosclerotic disease. In conclusion, the present study provided some support for the idea that CQC may offer a therapeutic strategy for the pathogenesis of Ang-II-related vascular diseases such as hypertension or atherosclerosis. It may also serve as a promising cardiovascular antiproliferative or antimigratory agent. Whether CQC acts on more upstream signal pathways beyond protein kinase or ROS requires further study.

\section{Funding}

National Research Institute of Chinese Medicine (NRICM97DBCMR-01), Taipei, Taiwan, ROC.

\section{References}

[1] K. M. Schmidt-Ott, S. Kagiyama, and M. I. Phillips, "The multiple actions of angiotensin II in atherosclerosis," Regulatory Peptides, vol. 93, no. 1-3, pp. 65-77, 2000. 
[2] M. B. Marrero, B. Schieffer, B. Li, J. Sun, J. B. Harp, and B. N. Ling, "Role of Janus kinase/signal transducer and activator of transcription and mitogen-activated protein kinase cascades in angiotensin II- and platelet-derived growth factor-induced vascular smooth muscle cell proliferation," Journal of Biological Chemistry, vol. 272, no. 39, pp. 24684-24690, 1997.

[3] P. N. Seshiah, D. S. Weber, P. Rocic, L. Valppu, Y. Taniyama, and K. K. Griendling, "Angiotensin II stimulation of NAD(P)H oxidase activity: upstream mediators," Circulation Research, vol. 91, no. 5, pp. 406-413, 2002.

[4] L. Saward and P. Zahradkas, "Angiotensin II activates phosphatidylinositol 3-kinase in vascular smooth muscle cells," Circulation Research, vol. 81, no. 2, pp. 249-257, 1997.

[5] R. Katso, K. Okkenhaug, K. Ahmadi, S. White, J. Timms, and M. D. Waterfield, "Cellular function of phosphoinositide 3Kinase: implications for development, immunity, homeostasis, and cancer," Annual Review of Cell and Developmental Biology, vol. 17, pp. 615-675, 2001.

[6] K. Shigematsu, H. Koyama, N. E. Olson, A. Cho, and M. A. Reidy, "Phosphatidylinositol 3-kinase signaling is important for smooth muscle cell replication after arterial injury," Arteriosclerosis, Thrombosis, and Vascular Biology, vol. 20, no. 11, pp. 2373-2378, 2000.

[7] C. Dugourd, M. Gervais, P. Corvol, and C. Monnot, "Akt is a major downstream target of PI3-kinase involved in angiotensin II-induced proliferation," Hypertension, vol. 41, no. 4, pp. 882-890, 2003.

[8] P. S. Ray, G. Maulik, G. A. Cordis, A. A. E. Bertelli, A. Bertelli, and D. K. Das, "The red wine antioxidant resveratrol protects isolated rat hearts from ischemia reperfusion injury," Free Radical Biology and Medicine, vol. 27, no. 1-2, pp. 160-169, 1999.

[9] D. F. Fitzpatrick, S. L. Hirschfield, and R. G. Coffey, "Endothelium-dependent vasorelaxing activity of wine and other grape products," American Journal of Physiology, vol. 265, no. 2, pp. H774-H778, 1993.

[10] H.-H. Chao, S.-H. Juan, J.-C. Liu et al., "Resveratrol inhibits angiotensin II-induced endothelin-1 gene expression and subsequent proliferation in rat aortic smooth muscle cells," European Journal of Pharmacology, vol. 515, no. 1-3, pp. 1-9, 2005.

[11] C. C. Chen, B. L. Wei, S. H. Loke, and W. F. Chiou, "3,4Di-O-Caffeoylquinic acid protects endothelial cells against oxidative stress and restores endothelium-dependent vasodilatation," Journal of Chinese Medicine, vol. 18, pp. 89-100, 2007 (Chinese).

[12] M.-J. Don, J.-F. Liao, L.-Y. Lin, and W.-F. Chiou, "Cryptotanshinone inhibits chemotactic migration in macrophages through negative regulation of the PI3K signaling pathway," British Journal of Pharmacology, vol. 151, no. 5, pp. 638-646, 2007.

[13] W.-F. Chiou, H.-R. Tsai, L.-M. Yang, and W.-J. Tsai, “C5a differentially stimulates the ERK1/2 and p38 MAPK phosphorylation through independent signaling pathways to induced chemotactic migration in RAW264.7 macrophages," International Immunopharmacology, vol. 4, no. 10-11, pp. 1329-1341, 2004.

[14] F. Contreras, M. A. de la Parte, J. Cabrera, N. Ospino, Z. H. Israili, and M. Velasco, "Role of angiotensin II AT1 receptor blockers in the treatment of arterial hypertension," American journal of therapeutics, vol. 10, no. 6, pp. 401-408, 2003.

[15] G. Jia, G. Cheng, D. M. Gangahar, and D. K. Agrawal, "Insulinlike growth factor- 1 and TNF- $\alpha$ regulate autophagy through c-jun N-terminal kinase and Akt pathways in human atherosclerotic vascular smooth cells," Immunology and Cell Biology, vol. 84, no. 5, pp. 448-454, 2006.

[16] S. R. Datta, A. Brunet, and M. E. Greenberg, "Cellular survival: a play in three akts," Genes and Development, vol. 13, no. 22, pp. 2905-2927, 1999.

[17] T. Takahashi, T. Taniguchi, H. Konishi, U. Kikkawa, Y. Ishikawa, and M. Yokoyama, "Activation of Akt/protein kinase B after stimulation with angiotensin II in vascular smooth muscle cells," American Journal of Physiology, vol. 276, no. 6, pp. H1927-H1934, 1999.

[18] S. Eguchi, H. Iwasaki, H. Ueno et al., "Intracellular signaling of angiotensin II-induced p70 S6 kinase phosphorylation at Ser411 in vascular smooth muscle cells. Possible requirement of epidermal growth factor receptor, Ras, extracellular signalregulated kinase, and AKT," Journal of Biological Chemistry, vol. 274, no. 52, pp. 36843-36851, 1999.

[19] W. T. Gerthoffer, "Mechanisms of vascular smooth muscle cell migration," Circulation Research, vol. 100, no. 5, pp. 607-621, 2007.

[20] A. Pflueger, A. J. Croatt, T. E. Peterson et al., "The hyperbilirubinemic Gunn rat is resistant to the pressor effects of angiotensin II," American Journal of Physiology, vol. 288, no. 3, pp. F552-F558, 2005.

[21] C. Viedt, U. Soto, H. I. Krieger-Brauer et al., "Differential activation of mitogen-activated protein kinases in smooth muscle cells by angiotensin II: involvement of p22phox and reactive oxygen species," Arteriosclerosis, Thrombosis, and Vascular Biology, vol. 20, no. 4, pp. 940-948, 2000.

[22] Y. Nakajima, M. Shimazawa, S. Mishima, and H. Hara, "Water extract of propolis and its main constituents, caffeoylquinic acid derivatives, exert neuroprotective effects via antioxidant actions," Life Sciences, vol. 80, no. 4, pp. 370-377, 2007.

[23] Y. Inokuchi, M. Shimazawa, Y. Nakajima, S. Suemori, S. Mishima, and H. Hara, "Brazilian green propolis protects against retinal damage in vitro and in vivo," Evidence-Based Complementary and Alternative Medicine, vol. 3, no. 1, pp. 71-77, 2006.

[24] M. D. dos Santos, L. Gobbo-Neto, L. Albarella, G. E. Petto de Souza, and N. P. Lopes, "Analgesic activity of di-caffeoylquinic acids from roots of Lychnophora ericoides (Arnica da serra)," Journal of Ethnopharmacology, vol. 96, no. 3, pp. 545-549, 2005.

[25] J. S. Tang, H. Gao, C. X. Wang et al., "Antioxidative phenylpropanoid-substituted epicatechin glycosides from Parabarium huaitingii," Planta Medica, vol. 75, no. 15, pp. 1586-1590, 2009.

[26] I. Dini, G. C. Tenore, and A. Dini, "New polyphenol derivative in Ipomoea batatas tubers and its antioxidant activity," Journal of Agricultural and Food Chemistry, vol. 54, no. 23, pp. 87338737, 2006.

[27] T. M. Hung, M. Na, P. T. Thuong et al., "Antioxidant activity of caffeoyl quinic acid derivatives from the roots of Dipsacus asper Wall," Journal of Ethnopharmacology, vol. 108, no. 2, pp. 188-192, 2006. 


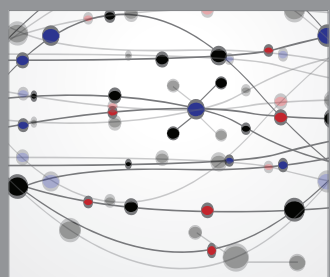

The Scientific World Journal
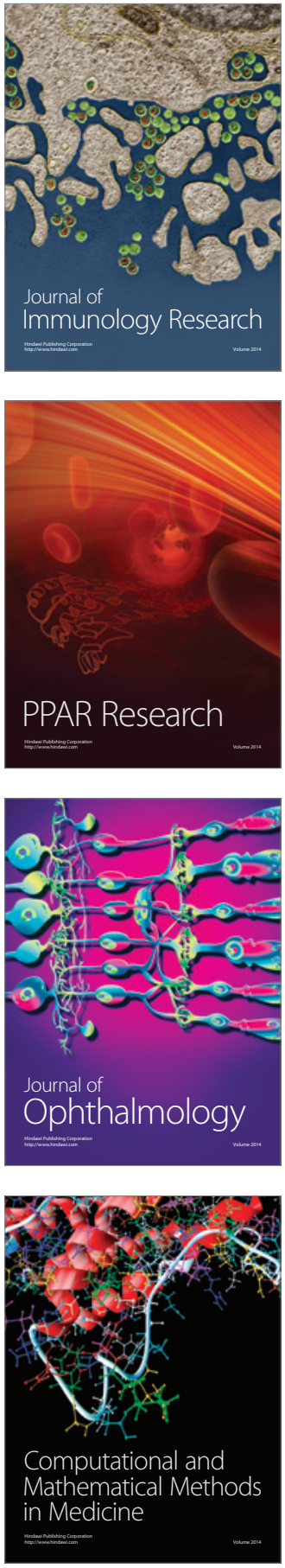

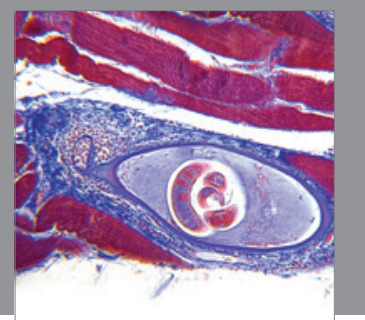

Gastroenterology

Research and Practice
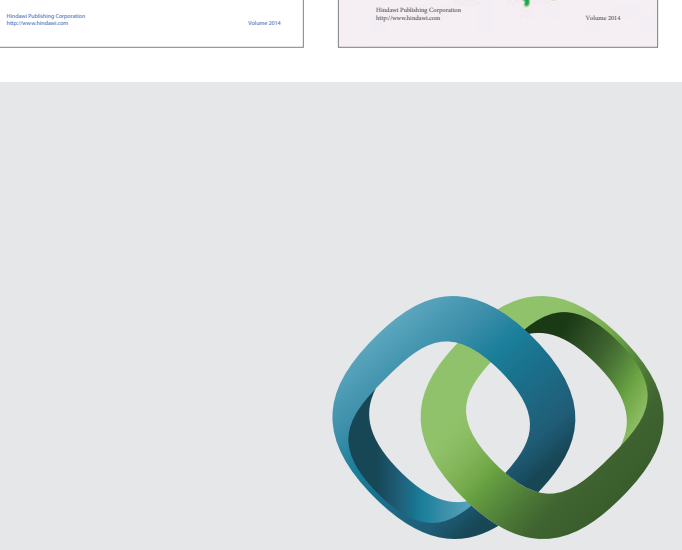

\section{Hindawi}

Submit your manuscripts at

http://www.hindawi.com
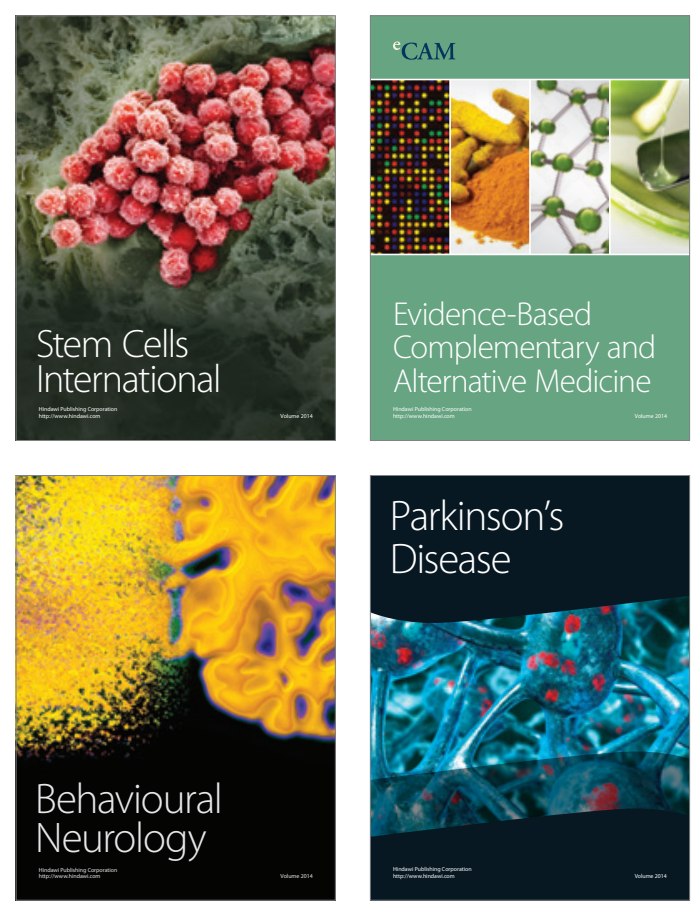

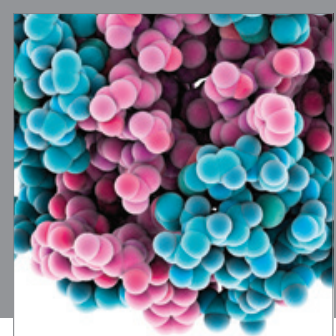

Journal of
Diabetes Research

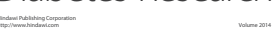

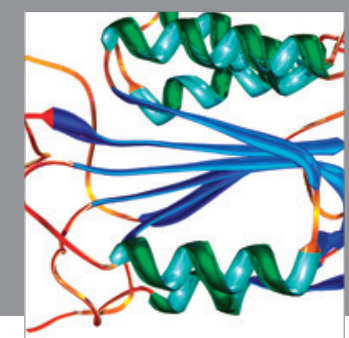

Disease Markers
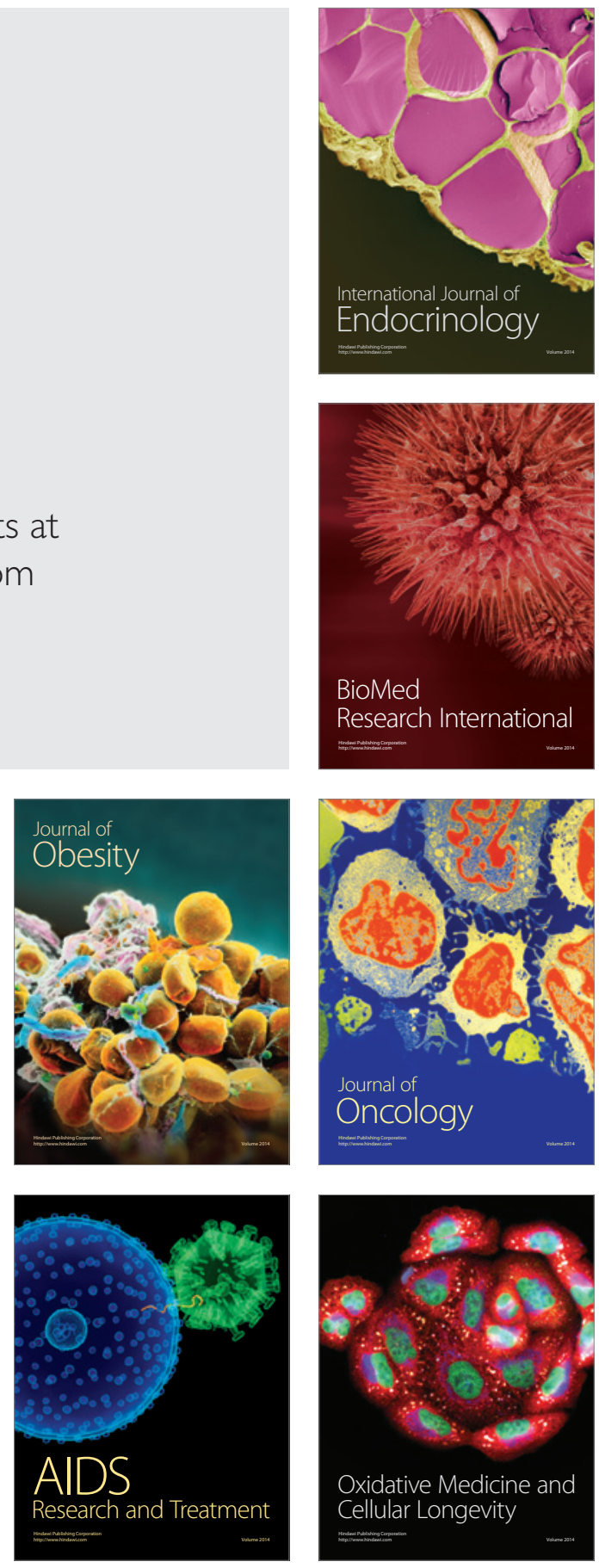